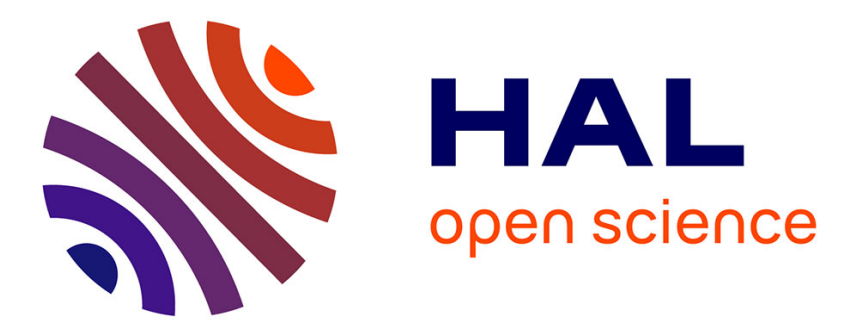

\title{
Sur le rôle respectif de la rugosité des interfaces et de l'inhomogénéité structurale dans la diffusion optique par une couche mince
}

\author{
P. Croce, L. Prod'Homme
}

\section{- To cite this version:}

P. Croce, L. Prod'Homme. Sur le rôle respectif de la rugosité des interfaces et de l'inhomogénéité structurale dans la diffusion optique par une couche mince. Revue de Physique Appliquée, 1977, 12 (10), pp.1641-1648. 10.1051/rphysap:0197700120100164100 . jpa-00244383

HAL Id: jpa-00244383

https://hal.science/jpa-00244383

Submitted on 1 Jan 1977

HAL is a multi-disciplinary open access archive for the deposit and dissemination of scientific research documents, whether they are published or not. The documents may come from teaching and research institutions in France or abroad, or from public or private research centers.
L'archive ouverte pluridisciplinaire HAL, est destinée au dépôt et à la diffusion de documents scientifiques de niveau recherche, publiés ou non, émanant des établissements d'enseignement et de recherche français ou étrangers, des laboratoires publics ou privés. 


\title{
SUR LE RÔLE RESPECTIF DE LA RUGOSITE DES INTERFACES ET DE L'INHOMOGÉNÉITÉ STRUCTURALE DANS LA DIFFUSION OPTIQUE PAR UNE COUCHE MINCE
}

\author{
P. CROCE et L. PROD'HOMME \\ Institut d'Optique, Bât. 503, Laboratoire associé au C.N.R.S. \\ Université Paris XI, B.P. 43, 91406 Orsay Cedex, France
}

(Reçu le $1^{\text {er }}$ avril 1977, accepté le 22 juin 1977)

\begin{abstract}
Résumé. - A l'aide d'une technique mathématique qui permet de calculer la répartition angulaire de l'intensité lumineuse diffusée par une couche mince, on montre qu'il est possible, grâce aux effets de polarisation de la lumière, de reconnaître l'origine de la diffusion en faisant quelques hypothèses simplificatrices sur la nature des défauts internes et externes à la couche. Les indicatrices de diffusion calculées pour une couche d'oxyde de titane, en tenant compte uniquement des défauts externes, sont en accord satisfaisant avec les résultats expérimentaux. Ceci met en évidence le rôle prépondérant de la rugosité des interfaces dans le processus de diffụsion observé.
\end{abstract}

Abstract. - We use a mathematical treatment specially designed for computing the angular dependence of light scattered from a thin film to show that, because of light polarization effects, it is possible to determine the origin of scattering, when a few simplifying assumptions as to the nature of both internal and external defects of the film are made.

The scattering indices computed for $\mathrm{a} \mathrm{TiO}_{2}$ thin film, are in good agreement with experimental results, when only external defects are taken into account. This shows the dominant role played by interfacial roughness in the observed scattering process.

1. Introduction. - L'étude de la diffusion optique par les couches minces présente un grand intérêt pratique, car dans les empilements de couches diélectriques servant de filtre ou de miroir pour les interféromètres Pérot-Fabry, la lumière perdue par diffusion est une des causes essentielles qui limitent les performances du système. C'est ce qu'avait déjà signalé Giacomo [1] dans une étude détaillée des couches multidiélectriques. Plus tard par microscopie électronique Koppelmann [2] puis Pearson [3] ont effectivement observé des défauts anisotropes à l'intérieur de couches obtenues par évaporation à vitesse rapide. Juranek [4] considère la mise en évidence de la lumière diffusée par le volume d'une couche comme une opération nécessaire, mais difficile. Enfin récemment, Günther et al. [5] ont étudié la répartition spatiale du flux diffusé par une couche multidiélectrique et ils ont expliqué le résultat obtenu par la superposition d'un effet de rugosité superficielle et d'un effet d'inhomogénéité structurale.

Dans une étude sur la nature des surfaces de verre poli [6], nous avons constaté que la diffusion optique peut s'expliquer uniquement par les seules irrégularités de la surface, sans qu'il soit nécessaire de tenir compte des défauts internes du verre. Mais la méthode utilisée pour traiter ce problème peut s'appliquer aussi au cas d'une couche mince déposée sur un substrat et nous allons montrer que dans certains cas et avec un minimum d'hypothèses naturelles on peut espérer séparer les contributions relatives aux deux origines distinctes de la diffusion que sont. d'une part les irrégularités géométriques des interfaces et d'autre part les défauts d'homogénéité structurale de la couche. L'étude en sera d'ailleurs facilitée par les informations obtenues par la réflexion spéculaire ou diffuse des rayons $\mathrm{X}$ qui renseignent sur l'épaisseur de la couche, sur la rugosité des interfaces et sur les facteurs de corrélation [7]. On supposera dans ce travail que la couche seule peut être diffusante dans la masse, à l'exclusion de son support.

2. Base théorique. - 2.1. PRINCIPE De LA MÉTHOde. - Pour établir les formules nécessaires à la discussion nous rappellerons ici le principe de la méthode proposée.

Considérons un milieu indéfini $\mathrm{R}$ limité par des surfaces rugueuses et dans lequel l'indice de réfraction est une fonction de l'espace $n_{\mathrm{R}}(\mathbf{r})$ où $\mathbf{r}$ désigne un point quelconque dans un système de coordonnées d'origine $\mathrm{O}$ qui sera précisé plus loin. 
Sous l'influence d'un champ électrique incident provenant d'une source $S$ éloignée, il apparaît dans ce milieu un champ électrique d'amplitude $\mathbf{E}_{\mathbf{R}}(\mathbf{r})$.

Ce champ crée une polarisation du milieu telle que, exprimée en C.G.S., on ait :

$$
\mathbf{P}_{\mathbf{R}}(\mathbf{r})=\frac{1}{4 \pi}\left[n_{\mathbf{R}}^{2}(\mathbf{r})-1\right] \mathbf{E}_{\mathbf{R}}(\mathbf{r}) \text {. }
$$

Or, en raison des irrégularités de la surface et de l'inhomogénéité de la matière, il est très difficile de connaître exactement $\mathbf{E}_{\mathbf{R}}(\mathbf{r})$. Toutefois, supposons que le milieu réel $\mathrm{R}$ soit très voisin d'un milieu idéal $\mathrm{I}$ où le champ induit en tout point par une source donnée est facile à déterminer, la polarisation acquise par ce milieu idéal possédant une répartition $n_{\mathrm{I}}(\mathbf{r})$ de l'indice de réfraction est égale, lorsqu'on lui impose le champ réel, à :

$$
\mathbf{P}_{\mathrm{I}}(\mathbf{r})=\frac{1}{4 \pi}\left[n_{\mathrm{I}}^{2}(\mathbf{r})-1\right] \mathbf{E}_{\mathbf{R}}(\mathbf{r}) .
$$

Nous allons voir que c'est précisément à la différence entre ces deux polarisations qu'on peut attribuer la diffusion optique, c'est-à-dire à :

$$
\Delta \mathbf{P}(\mathbf{r})=\frac{1}{4 \pi}\left[n_{\mathbf{R}}^{2}(\mathbf{r})-n_{\mathbf{I}}^{2}(\mathbf{r})\right] \mathbf{E}_{\mathbf{R}}(\mathbf{r}) .
$$

Nous considérons que cette polarisation est due à des dipôles fictifs n'ayant aucune interaction entre eux. Or, d'après les lois de Maxwell, pour une source extérieure donnée et pour une répartition de polarisation donnée, il n'existe qu'une seule répartition des champs. On retrouvera donc la répartition réelle du champ électrique dans le milieu réel en remplaçant ce dernier par un milieu artificiel obtenu en ajoutant les dipôles précédents au milieu idéal dans lequel ils rayonnent. L'emploi d'un cas idéal où les champs sont faciles à calculer par la loi du rayonnement à grande distance va nous permettre d'évaluer directement la valeur du champ diffusé au loin. Il suffit d'appliquer le principe de la réciprocité des champs énoncé par Lorentz : appelons $\mathbf{E}_{\mathbf{I}}(\mathbf{S}, \mathbf{r})$ le champ idéal créé par la source $S$ au point $\mathbf{r}$ (l'origine $O$ est située sur le plan idéal $\mathbf{P})$ et $\mathbf{E}_{\mathrm{I}}(\mathrm{D}, \mathbf{r})$ le champ idéal créé en ce même point $\mathbf{r}$ par un dipôle accessoire unitaire placé près du détecteur $\mathrm{D}$ et orienté normalement à $\mathrm{OD}$ (Fig. 1). Ce dipôle sera disposé dans la direction selon laquelle on veut connaitre la composante du champ. Ainsi la composante du champ diffusé par unité de volume s'exprime par le produit scalaire des deux vecteurs :

$$
\Delta \mathbf{P}(\mathbf{r}) . \mathbf{E}_{\mathbf{I}}(\mathrm{D}, \mathbf{r}) .
$$

L'amplitude totale de la composante cherchée correspondant au volume $V$, éclairé par l'émetteur et vu par le récepteur, est donc telle que :

$$
E_{\text {diff }}=\frac{1}{4 \pi} \int_{v}\left[n_{\mathbf{R}}^{2}(\mathbf{r})-n_{\mathrm{I}}^{2}(\mathbf{r})\right] \mathbf{E}_{\mathbf{R}}(\mathbf{r}) \cdot \mathbf{E}_{\mathbf{I}}(\mathrm{D}, \mathbf{r}) \mathrm{d} V
$$

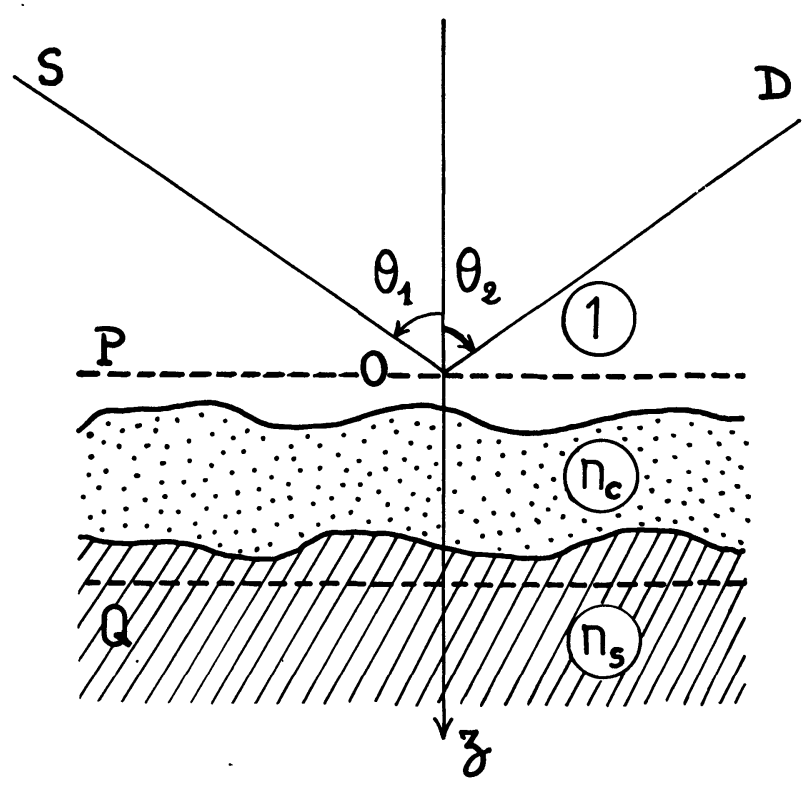

Fig. 1. - Disposition des plans idéaux $\mathrm{P}$ et $\mathrm{Q}$ par rapport à la couche réelle. ( $\mathrm{S}$, côté source; $\mathrm{D}$, côté détecteur).

[Location of ideal planes $P$ and $Q$ with respect to the real film (S, source side ; D, detector side).]

Cette expression est rigoureuse, mais elle n'est utilisable que si une approximation de la répartition réelle $\mathbf{E}_{\mathbf{R}}(\mathbf{r})$ peut facilement être évaluée à partir de la répartition idéale $\mathbf{E}_{I}(\mathbf{r})$. Ce sera le cas si l'on convient de faire certaines hypothèses simplificatrices sur la nature des défauts. Ainsi pour les défauts d'homogénéité structurale on se limitera soit à une faible variation de l'indice de réfraction à travers le volume éclairé, soit à de fortes variations locales dans des volumes élémentaires petits devant la longueur d'onde et qui dans leur ensemble ne constituent qu'une faible part du volume considéré. Pour les défauts des interfaces il faudra envisager uniquement des irrégularités de faible hauteur devant la longueur d'onde et une pente généralement douce.

Dans ces conditions le champ réel $\mathbf{E}_{\mathbf{R}}(\mathbf{r})$ se déduit linéairement du champ idéal $\mathbf{E}_{\mathrm{I}}(\mathbf{S}, \mathbf{r})$ par l'intermédiaire d'un opérateur linéaire de polarisation $\mathbf{E}(\mathbf{r})$ reliant les composantes du champ excitateur idéal à celles de la polarisation $\Pi(\mathbf{r})$. On a :

$$
\frac{1}{4 \pi}\left[n_{\mathbf{R}}^{2}(\mathbf{r})-n_{\mathbf{I}}^{2}(\mathbf{r})\right] \mathbf{E}_{\mathbf{R}}(\mathbf{r})=\Pi(\mathbf{r})\left[\mathbf{E}_{\mathbf{I}}(\mathbf{S}, \mathbf{r})\right]
$$

Il suffit d'ailleurs de connaître le champ excitateur avec une approximation d'ordre zéro pour calculer le champ diffusé au premier ordre. L'amplitude diffusée s'exprime maintenant en fonction de deux champs idéaux :

$$
E_{\text {diff }}=\int_{v} \mathbf{E}_{\mathrm{I}}(\mathbf{D}, \mathbf{r}) \cdot \Pi(\mathbf{r})\left[\mathbf{E}_{\mathrm{I}}(\mathbf{S}, \mathbf{r})\right] \mathrm{d} V .
$$

2.2. Diffuseurs DANS LA MASSE ET DIFFUSEURS AUX INTERFACES. - Cette expression générale peut s'appliquer aussi bien aux défauts diffusant dans la masse, 
qu'aux défauts diffusant aux interfaces. C'est l'opérateur $\Pi(\mathbf{r})$ qui permet de faire la distinction.

2.2.1. - Pour les défauts d'homogénéité structurale nous examinerons seulement le cas très simple où les diffuseurs dans la masse possèdent une disposition isotrope et une structure voisine de la symétrie sphérique. L'opérateur $\Pi(\mathbf{r})$, ou plus généralement sa moyenne prise dans un volume petit devant la longueur d'onde, peut alors être identifié à un opérateur unité $\mathbf{U}$ multiplié par un nombre aléatoire stationnaire $\mu(\mathbf{r})$ caractérisant le milieu en chaque point, puisque :

$$
\boldsymbol{\Pi}(\mathbf{r})=\mu(\mathbf{r}) \mathbf{U} .
$$

Le champ diffusé par un élément de volume $\mathrm{d} V$ est donc proportionnel à :

$$
\mu(\mathbf{r}) \mathbf{E}_{\mathrm{I}}(\mathrm{S}, \mathbf{r}) . \mathbf{E}_{\mathrm{I}}(\mathrm{D}, \mathbf{r}) \text {. }
$$

Étendue au volume $V$ l'intégrale (5) devient :

$$
E_{\text {diff }}=\int_{v} \mu(\mathbf{r}) \mathbf{E}_{\mathrm{I}}(\mathbf{S}, \mathbf{r}) \cdot \mathbf{E}_{\mathrm{I}}(\mathrm{D}, \mathbf{r}) \mathrm{d} V .
$$

$$
\Pi_{\mathrm{v}}=\frac{1}{4 \pi}\left(1-n_{\mathrm{c}}^{2}\right)\left[\begin{array}{lll}
1 & 0 & 0 \\
0 & 1 & 0 \\
0 & 0 & 1 / n_{\mathrm{c}}^{2}
\end{array}\right]
$$

et $\quad \Pi_{\mathrm{s}}=\frac{1}{4 \pi}\left(n_{\mathrm{s}}^{2}-n_{\mathrm{c}}^{2}\right)\left[\begin{array}{lll}1 & 0 & 0 \\ 0 & 1 & 0 \\ 0 & 0 & \frac{n_{\mathrm{s}}^{2}}{n_{\mathrm{c}}^{2}}\end{array}\right]$

L'intégrale de la formule générale (5) porte ainsi sur deux volumes petits compris entre la couche et les plans $\mathbf{P}$ et $\mathbf{Q}$. On remplacera dans cette formule les champs $\mathbf{E}_{\mathrm{I}}(\mathrm{D}, \mathbf{r})$ et $\mathbf{E}_{\mathrm{l}}(\mathbf{S}, \mathbf{r})$ par $\mathbf{E}_{\mathrm{d}}\left(\boldsymbol{\theta}_{2}, \mathbf{r}\right)$ et $\mathbf{E}_{\mathbf{i}}(\boldsymbol{\theta}, \mathbf{r})$. Ce sont les champs créés dans le milieu idéal sous l'influence d'ondes primaires qui proviennent respectivement des directions $\theta_{1}$ côté source et $\theta_{2}$ côté détecteur et dont l'amplitude est égale à 1 au point $O$ en l'absence de matière. Ils diffèrent des précédents par le terme principal du rayonnement d'un dipôle éloigné :

$$
A=\frac{4 \pi^{2}}{\lambda^{2} \rho}
$$

où $\lambda$ est la longueur d'onde dans le vide et $\rho$ la distance du dipôle à l'origine $\mathrm{O}$.

Relativement au plan $\mathbf{P}$ (côté vide) le produit scalaire écrit sous l'intégrale (5) prend donc, au facteur $A$ près, la valeur suivante :

$$
F_{\mathrm{v}}=\mathbf{E}_{\mathrm{d}}\left(\theta_{2}, 0\right) . \Pi_{\mathrm{v}}\left[\mathbf{E}_{\mathrm{i}}\left(\theta_{2}, 0\right)\right]
$$

et relativement au plan $\mathrm{Q}$ (côté support) ce même produit devient :

$$
F_{\mathrm{s}}=\mathbf{E}_{\mathrm{d}}\left(\theta_{2}, h\right) \cdot \Pi_{\mathrm{s}}\left[\mathbf{E}_{\mathrm{i}}\left(\theta_{2}, h\right)\right]
$$

où $E(\theta, z)$ désigne le champ au point de coordonnées $(0,0, z)$ et $h$ l'épaisseur de la couche.

Dans le cas général le calcul du champ diffusé résultant de ces deux contributions doit faire interve-
2.2.2. - Dans le cas de défauts aux interfaces nous examinerons seulement le cas particulier d'une couche mince dont l'indice de réfraction moyen est égal à $n_{\mathrm{c}}$ et qui est déposée sur un support d'indice $n_{\mathrm{s}}$.

En étendant quelque peu le raisonnement utilisé dans une étude précédente [6] les deux plans idéaux $P$ et $\mathbf{Q}$ seront placés dans un même milieu et extérieurement à la couche réelle de manière à ne faire intervenir les champs idéaux qu'à l'intérieur de la couche mince (Fig. 1). L'origine des coordonnées $O$ est sur le plan $\mathrm{P}$, les axes $\mathrm{O} x$ et $\mathrm{O} y$ sont dans ce plan et $\mathrm{O} z$ est dirigé selon la normale vers le support. On peut alors exprimer le champ réel à partir du champ idéal en admettant qu'il varie peu dans les volumes d'intégration et appliquer les lois de conservation des composantes tangentielles du champ électrique d'une part et normale de l'induction électrique d'autre part. Pour les deux interfaces vide-couche et supportcouche les deux opérateurs $\Pi_{v}$ et $\Pi_{s}$ seront donc, en prenant pour base des matrices les composantes des vecteurs selon $\mathrm{O} x, \mathrm{O} y, \mathrm{O} z$ :

nir la rugosité de chaque interface et le facteur de corrélation des deux profils. Nous ne développerons donc pas l'expression mathématique de ce champ; elle serait longue et sans application ici. Nous la donnerons seulement dans un cas particulier étudié au paragraphe suivant.

3. Application à la séparation des deux types de diffuseurs. - 3. 1. INTERPRÉTATION DES INDICATRICES DE DIFFusion. - Dans le cas général où une couche mince est susceptible de posséder les deux types de diffuseurs, l'amplitude du champ diffusé est donc en principe la somme de deux termes, l'un se rapportant aux défauts internes, l'autre aux défauts superficiels. Mais c'est le carré du module de cette somme qui est la quantité accessible à l'expérience dans les indicatrices de diffusion. L'expression de l'intensité diffusée devrait donc contenir des termes croisés provenant de l'intensité des deux champs de type différent. Or, tous les termes croisés sont nuls si l'on admet qu'il n'y a aucune corrélation latérale de la position des deux types de diffuseurs, c'est-à-dire aucune corrélation de la position des défauts internes et externes dans tout plan parallèle à la couche étudiée, étant entendu que celle-ci, a une épaisseur grande devant la hauteur des rugosités. Dans ces conditions les intensités mesurées sur les indicatrices correspondent à la somme des intensités provenant de l'un et de l'autre type de diffuseurs.

Ces considérations montrent qu'il doit être possible 
de reconnaître l'origine de la diffusion par une couche mince en comparant les indicatrices théoriques aux indicatrices expérimentales. En diffusion optique en particulier on pourra étudier le rapport $i_{\mathrm{s}} / i_{\mathrm{p}}$ des intensités diffusées en utilisant les deux polarisations $\mathrm{s}$ et $\mathrm{p}$ du champ excitateur.

Mais dans le cas de diffusion des rayons $\mathrm{X}$, il faut remarquer aussitôt que les matrices présentées plus haut ne sont pas influencées par la nature des diffuseurs, puisque les indices de réfraction sont voisins de 1 . Il est donc impossible avec nos hypothèses de séparer les deux origines de la diffusion par les indicatrices en $\mathrm{s}$ et $\mathrm{p}$. Par contre, les interférences qu'on peut observer entre les amplitudes diffractées par les deux interfaces de la couche, permettent d'accéder aux facteurs de corrélation des défauts entre ces interfaces. La méthode qui consiste à mesurer l'intensité diffusée à fréquence spatiale $K_{\mathrm{d}}$ constante en suivant la loi des réseaux a été appliquée à une couche déposée sur du verre et elle a mis en évidence des franges contrastées dont la position dépend nettement de l'épaisseur de la couche [9] (le vecteur $K_{\mathrm{d}}$ sera défini plus loin). Ceci prouve que les diffuseurs sont situés au voisinage des interfaces et qu'il existe une forte corrélation entre leurs défauts respectifs. $\left({ }^{1}\right)$. Il est très probable d'après ce que l'on sait de la structure des couches minces qu'il s'agit uniquement dans cet exemple d'une diffusion par les rugosités.

En diffusion optique il faut s'attendre au contraire à ce que les opérateurs soient fortement influencés par la nature des diffuseurs. Les indicatrices obtenues seront en outre différentes selon les polarisations $s$ ou p.

En ce qui concerne la nature des diffuseurs, notre étude se limite, comme il a été dit plus haut, à la diffusion isotrope de Rayleigh pour les défauts internes et à une rugosité douce pour les défauts superficiels. Une manière simple de s'assurer que ces conditions sont remplies consiste à vérifier que l'intensité de la vibration diffusée en quadrature de la vibration incidente est suffisamment faible pour chacune des deux combinaisons sp ou ps.

3. 2. CAlCUl DES INDICATRICES DE DIFFUSION. 3. 2. 1. Défauts d'homogénéité. - Pour calculer l'intensité diffusée nous appelons $L$ la luminance du faisceau laser excitateur et $s_{1}$ la section du diaphragme qui le limite. Si le faisceau diffusé n'est luimême limité que par un seul diaphragme de section $s_{2}$, l'intensité totale diffusée dans la surface $s_{2}$ est égale à :

$$
i=L \times \frac{s_{1}}{\cos \theta_{1}}\left(\frac{4 \pi^{2}}{\lambda^{2} \rho}\right)^{2} \times s_{2} \times \bar{\mu}^{2} \int_{0}^{h}\left[\mathbf{E}_{\mathbf{i}}\left(\theta_{1}\right) \cdot \mathbf{E}_{\mathrm{d}}\left(\theta_{2}\right)\right]^{2} \mathrm{~d} z
$$

où $\bar{\mu}^{2}=\frac{1}{v}\left|\int \mu(\mathbf{r}) \mathrm{d} v\right|^{2}$ représente la valeur quadra-

$1,52)$ et éclairée par une source $\lambda=0,6328 \mu \mathrm{m}$ sous une incidence $\theta_{1}=75^{\circ}$. La figure 2 représente les

tique moyenne du terme d'inhomogénéité.

On admet implicitement ici que le spectre de ces défauts est blanc; mais même si ce n'était pas le cas, l'erreur qui en résulterait serait faible, car dans une couche fortement réfringente les ondes se propagent toujours au voisinage de la normale quelle que soit l'incidence du rayon extérieur excitateur. Dans ces conditions les champs $\left|\mathbf{E}_{\mathrm{i}}\left(\boldsymbol{\theta}_{1}\right)\right|$ et $\left|\mathbf{E}_{\mathrm{d}}\left(\boldsymbol{\theta}_{2}\right)\right|$, considérés comme fonction de l'épaisseur, restent assez semblables quand $\theta_{1}$ et $\theta_{2}$ varient.

L'intégrale qui apparaît dans cette formule caractérise la géométrie d'une diffusion lumineuse par une tranche de matière inhomogène. La répartition angulaire correspondante est donnée par :

$$
\int_{0}^{h}\left[\mathbf{E}_{\mathrm{i}}\left(\theta_{1}\right) \cdot \mathbf{E}_{\mathrm{d}}\left(\theta_{2}\right)\right]^{2} \mathrm{~d} z=\alpha_{\mathrm{H}}^{2}\left(\theta_{1}, \theta_{2}\right) \times h .
$$

Son allure n'est pas modifiée par le facteur constant $\bar{\mu}^{2}$.

Le calcul a été fait pour une couche de $\mathrm{TiO}_{2}$ $\left(n_{\mathrm{c}}=2,7 ; e=610 \AA\right)$ déposée sur un verre $\left(n_{\mathrm{s}}=\right.$

(1) Le facteur de corrélation déterminé grâce aux rayons $\mathrm{X}$ porte sur des sections de la surface puisque l'appareil utilise des fentes. En diffusion optique le laser permet d'opérer avec des trous et le facteur de corrélation doit être légèrement supérieur, à fréquence spatiale égale, car il diminue quand cette fréquence augmente.

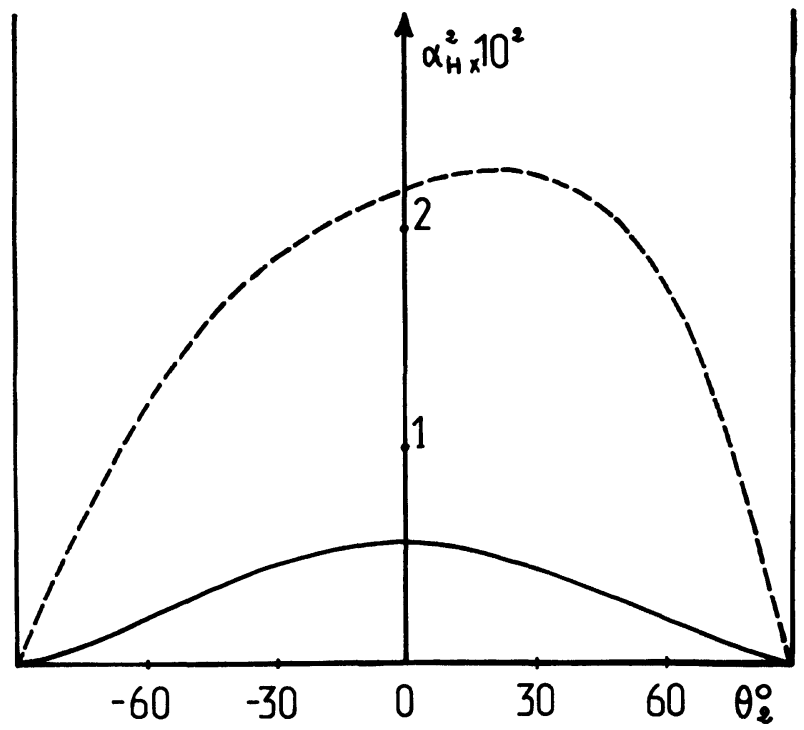

Fİ́. 2. - Fonctions $\alpha_{\mathrm{H}}^{2}\left(\theta_{1}, \theta_{2}\right)$ représentant les indicatrices d'une diffusion par les défauts d'homogénéité d'une couche mince éclairée sous un angle d'incidence $\theta_{1}=75^{\circ}$. Les angles $\theta_{2}$ positifs correspondent à la rétrodiffusion. (- polarisation $\mathrm{s} ; \ldots$. polarisation p).

$\left[\alpha_{\mathrm{H}}^{2}\right.$-functions related to the scattering process due to inhomogeneity of a thin film irradiated with angle of incidence $\theta_{1}=75^{\circ}$. Positive $\theta_{2}$-values refer to back-scattering. ( - s-polarization; ...... p-polarization).] 
fonctions $\alpha_{\mathrm{H}}^{2}\left(\theta_{1}, \theta_{2}\right)$ qui correspondent à un facteur près aux indicatrices en $\mathrm{s}$ et $\mathrm{p}$ de ce type de diffusion.

3. 2. 2. Défauts aux interfaces. - Pour calculer maintenant les indicatrices de diffusion par les rugosités des deux interfaces d'une couche mince, nous envisageons uniquement le cas particulier d'une corrélation parfaite. Ceci signifie que non seulement la transformée de la fonction d'autocorrélation des défauts, considérée dans sa moyenne sur une surface $S$ suffisamment étendue et qui définit habituellement le spectre de rugosité

$$
T^{2}\left(K_{\mathrm{d}}\right)=\frac{1}{S}\left|\int_{S} z \mathrm{e}^{2 \pi i K_{\mathrm{d}} z} \mathrm{~d} \sigma\right|^{2}
$$

avec $K_{\mathrm{d}}=\frac{1}{\lambda}\left(\sin \theta_{2}-\sin \theta_{1}\right)$, est rigoureusement la même pour les deux interfaces, mais aussi que ces derniers se déduisent l'un de l'autre par simple translation. Dans ces conditions les produits scalaires $F_{v}$ et $F_{s}$ relatifs à chaque interface contribuent simultanément à l'amplitude diffusée et, avec la convention
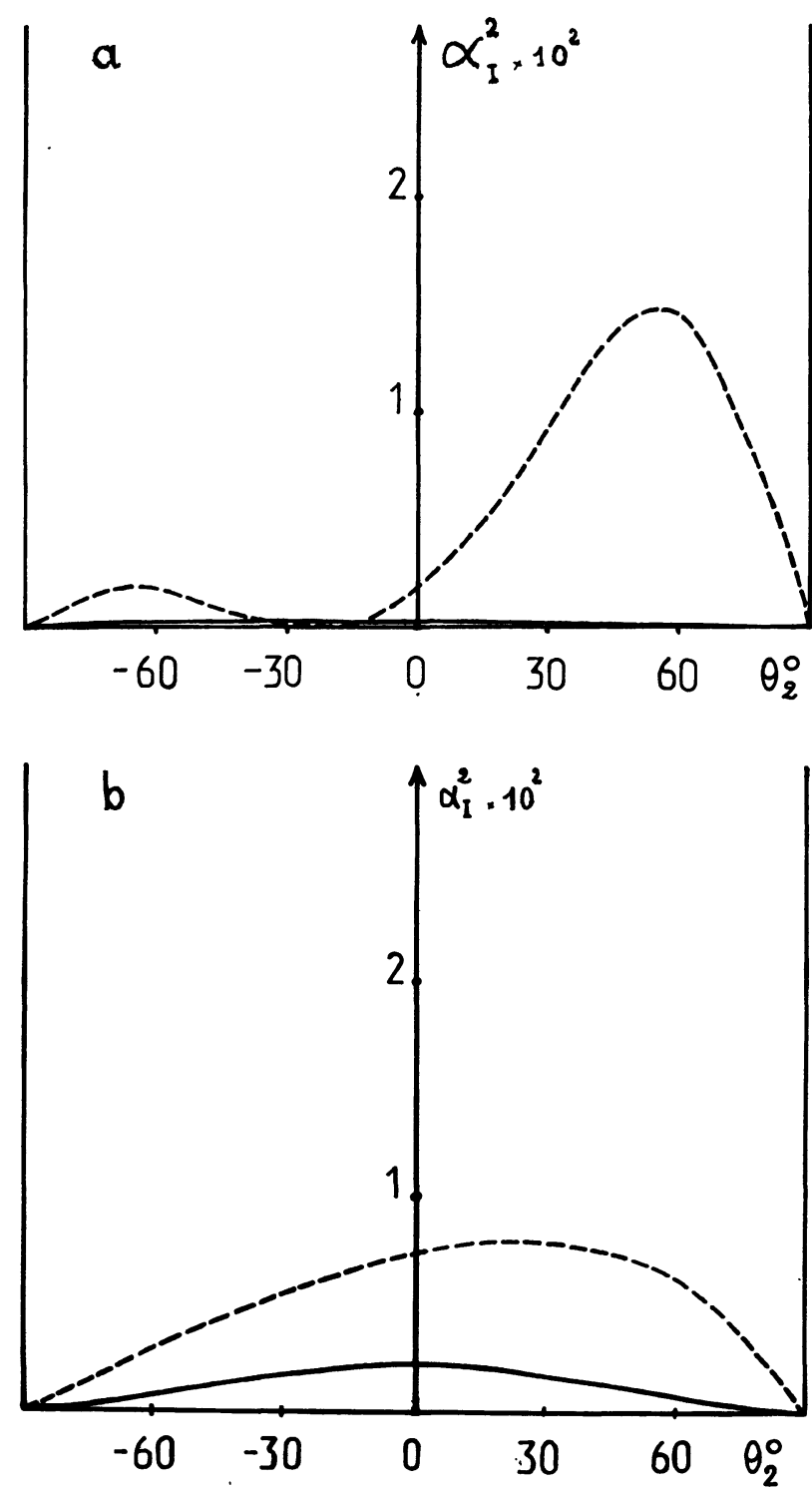
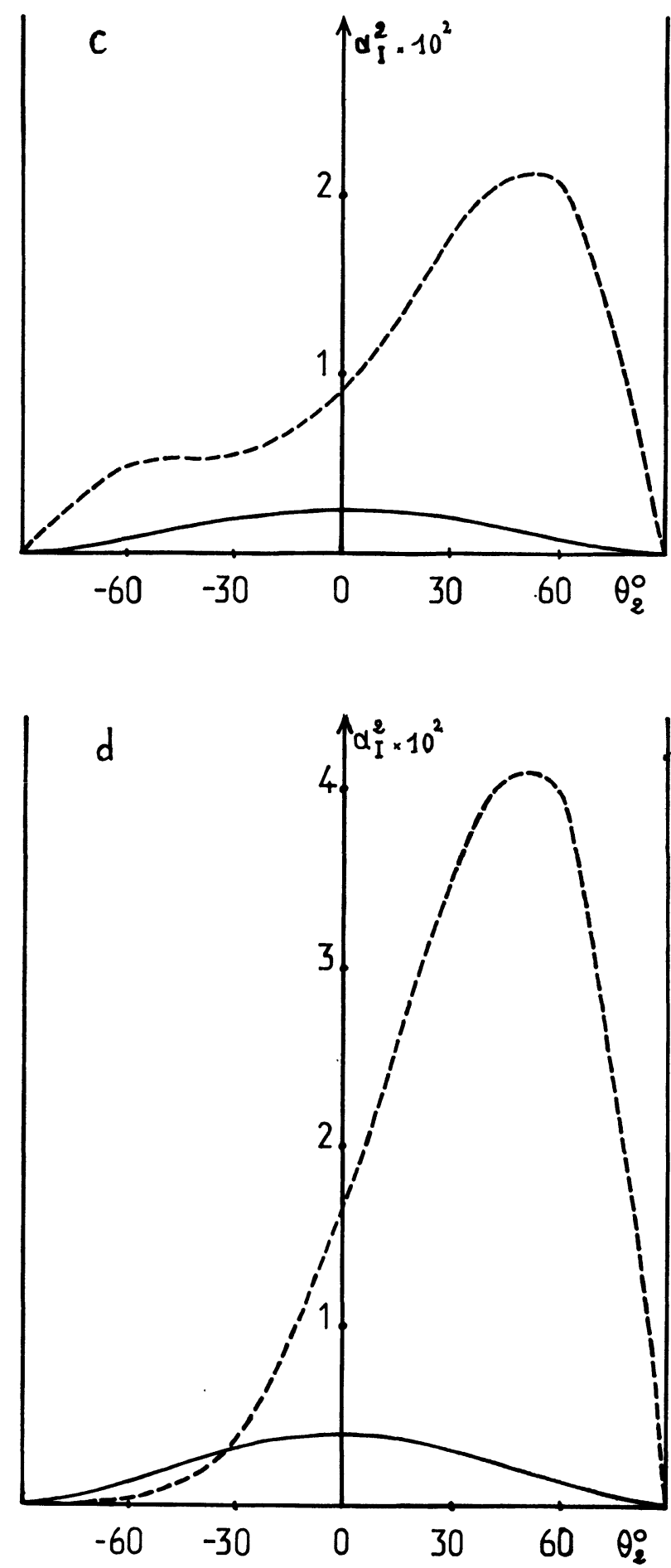

FIG. 3. - Fonctions $\alpha_{1}^{2}\left(\theta_{1}, \theta_{2}\right)$ caractérisant la diffusion par la rugosité des interfaces d'une couche mince éclairée sous un angle d'incidence $\theta_{1}=75^{\circ}$. Les angles $\theta_{2}$ positifs correspondent à la rétrodiffusion. ( - polarisation $s ;$..... polarisation $\mathrm{p}$ ). On a fait intervenir l'interface vide-couche en a, l'interface couche-support en $b$, les deux interfaces non corrélés en $c$ et les deux interfaces parfaitement corrélés en $d$.

$\left[\alpha_{1}^{2}\right.$-functions characterising the scattering process due to interface roughness for a thin film irradiated with angle of incidence $\theta_{1}=75^{\circ}$. Positive $\theta_{2}$-values refer to back-scattering. (- spolarization; ..... p-polarization). Taken into account are (a) the vacuum-film interface alone, (b) the film-substrate interface alone, (c) both interfaces non-correlated and (d) both interfaces perfectly correlated.] 
de signe qui résulte de la position des plans $\mathrm{P}$ et $\mathrm{Q}$, l'intensité diffusée par les irrégularités aux interfaces est exprimée par :

$i=L \times \frac{s_{1}}{\cos \theta_{1}}\left(\frac{4 \pi^{2}}{\lambda^{2} \rho}\right)^{2} \times s_{2} \times T^{2}\left(K_{\mathrm{d}}\right)\left|F_{\mathrm{v}}-F_{\mathrm{s}}\right|^{2}$

La fonction $\left|F_{\mathrm{v}}-F_{\mathrm{s}}\right|^{2}$ qui caractérise la géométrie d'une diffusion lumineuse par deux interfaces corrélés sera appelée $\alpha_{1}^{2}\left(\theta_{1}, \theta_{2}\right)$. Pour la même couche que cidessus placée dans les mêmes conditions, la figure 3 montre les répartitions calculées en $s$ et $p$ dans certains cas particuliers. Ainsi en 3a on a pris en compte seulement les rugosités de l'interface vide-couche, et la fonction $\alpha_{\mathrm{I}}^{2}$ se réduit à $\left|F_{\mathrm{v}}\right|^{2}$, en $3 \mathrm{~b}$ uniquement celles de l'interface couche-support, et $\alpha_{1}^{2}$ devient $\left|F_{\mathrm{s}}\right|^{2}$, en $3 \mathrm{c}$ on a additionné ces deux contributions en admettant l'absence de corrélation avec cependant le même spectre de rugosité pour chaque interface, de sorte que $\alpha_{1}^{2}=\left|F_{\mathrm{v}}\right|^{2}+\left|F_{\mathrm{s}}\right|^{2}$. Enfin la figure 3d représente le cas de la corrélation parfaite où $\alpha_{1}^{2}=$ $\left|F_{\mathrm{v}}-F_{\mathrm{s}}\right|^{2}$. L'aspect des courbes obtenues est très différent selon les cas.

Pour calculer l'intensité diffusée nous adopterons l'hypothèse de la corrélation parfaite qui est d'ailleurs proche de la valeur déduite d'une étude effectuée aux rayons $X$ [9]. Le spectre de rugosité qui intervient

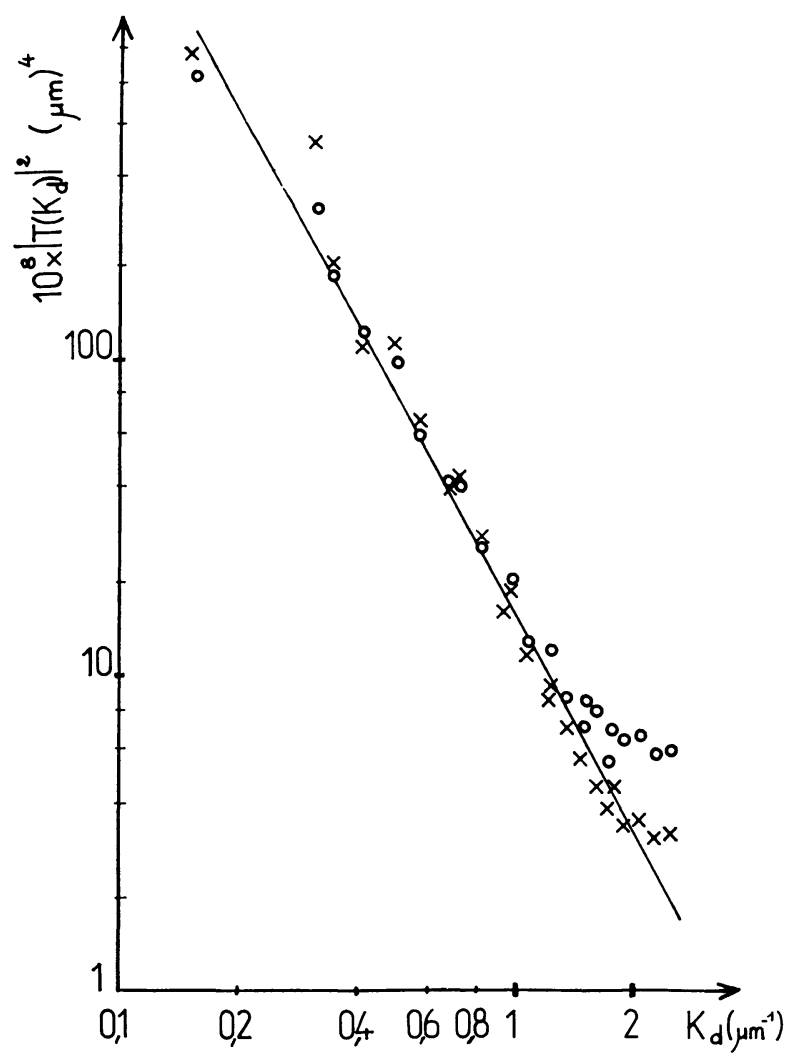

Fig. 4. - Spectre de rugosité de la surface d'un support nu déduit de plusieurs séries de mesures. ( $\bullet$, mesures en polarisation $\mathrm{s}$; $\mathrm{x}$, mesures en polarisation $\mathrm{p}$ )

[Roughness spectrum of an uncoated substrate surface as derived from a number of measurements made with both s-polarized (•) and p-polarized $(x)$ light.] dans ce calcul a été déterminé expérimentalement par diffusion optique de la surface d'un verre identique à celui du support de la couche. La figure 4 rassemble les résultats expérimentaux qui ont permis de connaître le spectre de rugosité (trait plein) le plus probable dans le domaine des fréquences spatiales comprises entre 0,2 et $2 \mathrm{~m} \mu^{-1}$.

Les indicatrices calculées pour trois angles d'incidence différents, c'est-à-dire $50^{\circ}, 60^{\circ}$ et $75^{\circ}$ sont représentées sur la figure 5 , en adoptant les constantes de l'appareil qui servira aux expériences. En particulier le produit $L s_{1}$ est exprimé par le courant photoélectrique du détecteur recevant directement le faisceau incident. L'intensité $i$ est donc mesurée en ampères.

3. 3. COMPARAISON DE LA THÉORIE À L'EXPÉRIENCE. - Les indicatrices de diffusion de la couche d'oxyde de titane décrite ci-dessus ont été obtenues grâce au montage goniométrique que nous avons décrit dans un article précédent [6]. Les intensités $i$ directement mesurées en nA sont rapportées sur les trois graphiques de la figure 5 , les points concernent la vibration s, et les croix la vibration $\mathrm{p}$. L'intensité diffusée résiduelle qui est due aux défauts dépolarisants et que l'on mesure quand analyseur et polariseur sont en quadrature se situe pour la couche examinée à un niveau très bas, c'est-à-dire $i_{\mathrm{sp}}=0,2 \mathrm{nA}$ et $i_{\mathrm{ps}}=0,3 \mathrm{nA}$ (le courant d'obscurité du détecteur est de l'ordre de $0,1 \mathrm{nA})$. Précisons que le produit $L s_{1}$ correspond à $0,8 \mathrm{~A}$, que $s_{2}=1 \mathrm{~mm}^{2}$ et $\rho=100 \mathrm{~mm}$.

L'accord satisfaisant qui apparaît entre les courbes calculées et les points de mesure met en évidence le rôle prépondérant des irrégularités aux interfaces dans le phénomène de diffusion lumineuse et confirme l'existence d'une forte corrélation entre les deux profils. Si cette couche de $\mathrm{TiO}_{2}$ avait une structure quelque peu inhomogène, il est probable qu'on constaterait un niveau général de diffusion sensiblement relevé par rapport à celui des indicatrices calculées. Dans le cas de diffusion vers l'avant $\left(\theta_{1}<0\right)$ l'importance de $\left|T\left(K_{\mathrm{d}}\right)\right|^{2}$ est telle que la diffusion par les interfaces est prépondérante bien que les fonctions $\alpha_{1}^{2}$ $\left(\theta_{1}, \theta_{2}\right)$ soient faibles. On ne pourrait donc détecter l'effet d'une diffusion par les défauts internes qu'en rétrodiffusion $\left(\theta_{1}>0\right)$, mais la précision des mesures est insuffisante pour déterminer la contribution d'une inhomogénéité structurale qui de toute façon doit être très faible.

4. Conclusion. - Ce premier essai qui concerne l'étude d'un cas particulièrement simple montre qu'il est possible de séparer les deux origines de la diffusion lumineuse par une couche mince, à condition bien entendu que les homogénéités responsables du phénomène restent dans les limites admises pour la validité de la théorie.

Le léger désaccord observé entre valeurs calculées et valeurs mesurées est peut-être dû à une corrélation imparfaite des rugosités aux deux interfaces, mais 

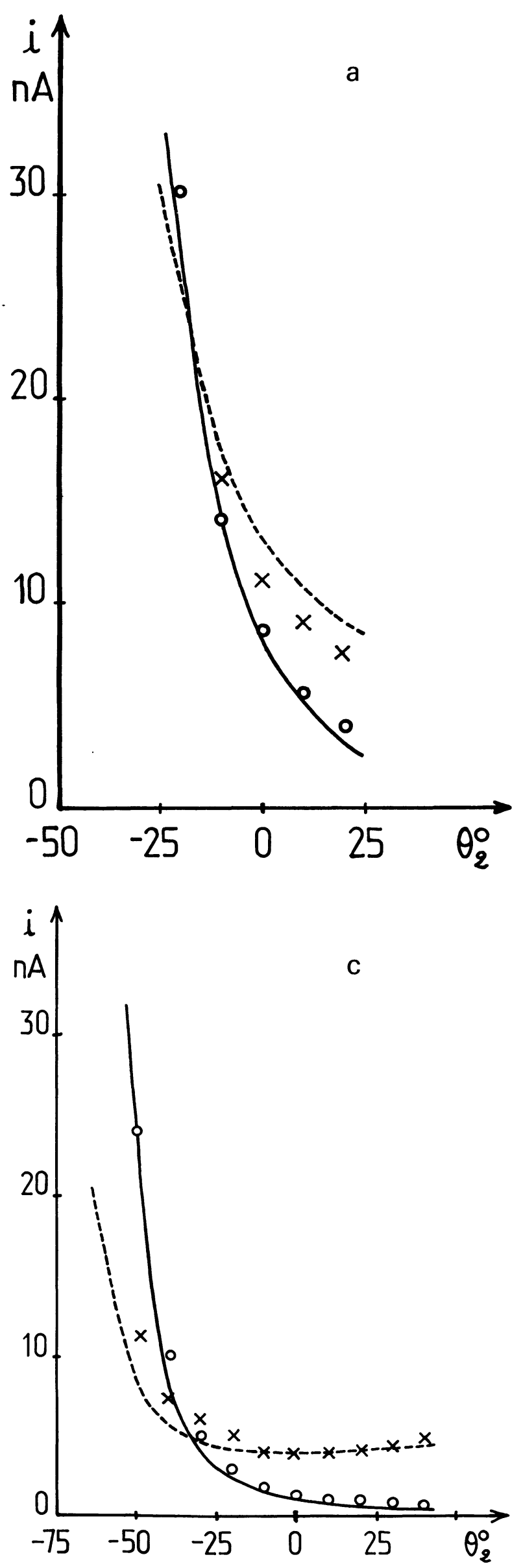

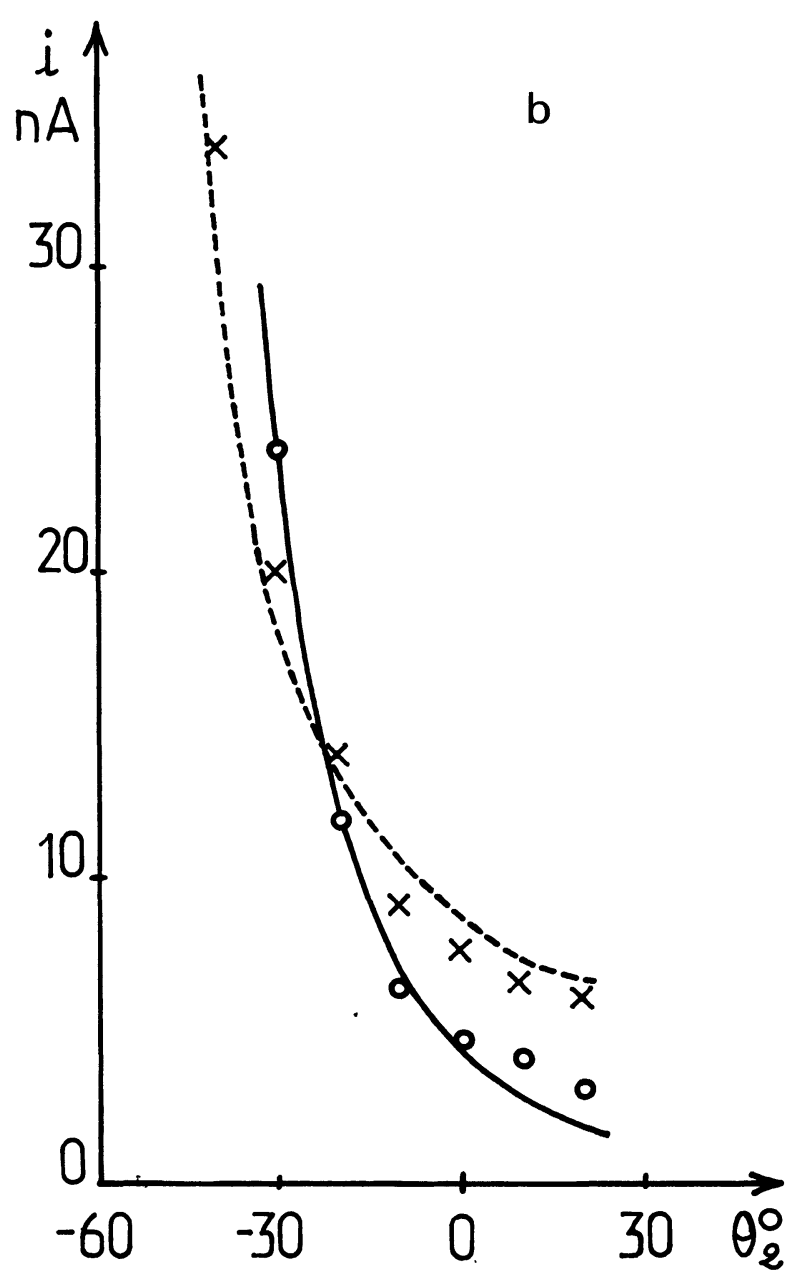

FIG. 5. - Indicatrices de diffusion par les interfaces rugueux d'une couche mince de $\mathrm{TiO}_{2}$ calculées à partir de la courbe tracée sur la figure 4 pour trois angles d'incidence différents du faisceau excitateur : $\theta_{1}=50^{\circ}$ en a, $60^{\circ}$ en b et $75^{\circ}$ en c. ( - polarisation s; .... polarisation $\mathrm{p}$ ). Les valeurs mesurées sont indiquées par • en polarisation $\mathrm{s}$ et par $\mathrm{x}$ en polarisation $\mathrm{p}$.

[Scattering indices due to the rough interfaces of a $\mathrm{TiO}_{2}$-film deposited on glass; they are computed from the curve drawn in figure 4 for three different angles of incidence: (a) $\theta_{1}=50^{\circ}$, (b) $\theta_{1}=60^{\circ}$ and (c) $\theta_{1}=75^{\circ}$. ( - s-polarization; ...... p-polarization). Measured values are shown for s-polarized $(\bullet)$ and p-polarized $(\mathrm{x})$ light.]

certainement plus encore aux conditions expérimentales qui réduisent notablement la précision des mesures. La présence de fines poussières à la surface des échantillons est un peu moins gênante dans ces expériences que dans l'étude des surfaces optiquement polies car le flux diffusé est nettement plus intense. Elle n'en constitue pas moins un obstacle à des mesures précises que nous espérons pouvoir réaliser bientôt en atmosphère dépoussiérée.

Remerciements. - Nous tenons à exprimer ici nos remerciements à Monsieur Névot pour les études effectuées à l'aide des rayons $\mathrm{X}$, et à Madame Valiergue pour la réalisation des couches. 


\section{Bibliographie}

[1] Giacomo P., Rev. Opt. Theor. Instrum. 35 (1956), 442.

[2] Koppelmann G., Optik 17 (1960) 416.

[3] Pearson J. M., Thin Solid Films 6 (1970) 349.

[4] JuraneK J., Z. Phys. 233 (1970) 324.

[5] Gunther K. H., Gruber H. L. et Pulker H. K., Thin Solid Films 34 (1976) 363.
[6] Croce P. et Prod'homme L., Nouv. Rev. Opt. 7 (1976) 121.

[7] Croce P. et Nevot L., Revue Phys. Appl. 11 (1976) 113.

[8] Croce P., Nouv. Rev. Opt. 8 (1977) 127.

[9] Nevot L., Thèse, Orsay (1977) à paraître. 